\title{
State Feedback Optimal Control with Singular Solution for a Class of Nonlinear Dynamics
}

\author{
Paolo Di Giamberardino ${ }^{1}$ and Daniela Iacoviello ${ }^{1,2}$ \\ ${ }^{1}$ Dept. Computer, Control and Management Engineering Antonio Ruberti, Sapienza University of Rome, Italy \\ ${ }^{2}$ Institute for Systems Analysis and Computer Science Antonio Ruberti, Rome, Italy
}

Keywords: Optimal Control, Singular Control, Costate Independent Singular Surface, SIR Epidemic Model.

\begin{abstract}
The paper studies the problem of determining the optimal control when singular arcs are present in the solution. In the general classical approach the expressions obtained depend on the state and the costate variables at the same time, so requiring a forward-backward integration for the computation of the control. In this paper, sufficient conditions on the dynamics structure are provided and discussed in order to have both the control and the switching function depending on the state only, so simplifying the computation avoiding the necessity of the backward integration. The approach has been validated on a classical SIR epidemic model.
\end{abstract}

\section{INTRODUCTION}

Optimal control theory provides the natural framework to solve control problems when contrasting goals are required with resource limitations. The design procedure can make use of the minimum principle, allowing the determination of the optimal control that, depending on the cost index and on the modeling, could be a bang-bang or a bang-singular-bang solution, (Athans and Falb, 1996; Hartl et al., 1995; Johnson and Gibson, 1963; Bryson and Ho, 1969). In particular, if the model as well as the cost index are linear in the control, the existence conditions of such kind of solutions can be explicitly determined. In the bang-bang solution the control assumes only the extreme values, whereas the singular one is obtained if the Hamiltonian does not depend on the control in an interval of positive measure. The extreme values assumed by the control depend on the sign of the switching function, whereas the existence of singular control is related to the possibility that this switching function is identically zero on an interval of finite length.

The determination of singular control, while it is easy from a theoretical point of view, is generally difficult to implement; the optimal control requires the solution of a non linear differential equations system in the state variables with initial conditions, and a non linear differential equations system in the costate variables with final conditions. Moreover, in general it is not easy the determination of the best control se- quence and the number of switching points, (Vossen, 2010; Fraser-Andrews, 1989).

In this paper, the determination of the optimal control of nonlinear systems is investigated referring to the case in which the input acts linearly both in the model and in the cost index, aiming at a constructive computing of the singular solution. This approach is then applied to a classical SIR epidemic problem, where $\mathrm{S}$ stands for the class of susceptible subjects, I for the class of infected patients and $\mathrm{R}$ for the class of the removed ones (Di Giamberardino and Iacoviello, 2017; Bakare et al., 2014). Optimal control for epidemic spread containment has been widely used in literature (Behncke, 2000; Di Giamberardino et al., 2018). In particular, the optimal singular control for a SIR epidemic model has been already studied in (Ledzewicz and Schattler, 2011; Ledzewicz et al., 2016), where the structure of singular control has been deeply investigated in presence of the double control, vaccination and medical treatment, showing that the latter can't be singular, whereas a singular regimen is expected for the optimal vaccination strategy. Differently from (Ledzewicz et al., 2016), in this paper a recovered subject could neither become susceptible, nor infected again. Therefore, it is possible to study the singular surface, that is the manifold over which the state variables move under the action of the singular control, if it exists. Facing epidemic spread control in the framework of optimal control theory is rather common for its capability of suggesting suitable scheduling of possible actions such as vaccination or 
quarantine or treatment, taking into account resource limitations. The paper is organized as follows; the optimal control problem is formulated in Section 2, describing the conditions and the constraints usually considered and giving the structure of the optimal solution as well as the conditions for the existence of both switches and singular arcs. Then, in Section 3 the class of dynamics considered is introduced and constructive conditions for the singular solutions are given for different formulations of the optimal problem. In Section 4 the case study describing the SIR epidemic spread is introduced to verify and highlight the effectiveness of the results.

\section{THE OPTIMAL CONTROL PROBLEM FORMULATION}

The optimal control problem here addressed is referred to the design of the control input $u$, for a given nonlinear dynamics of the form

$$
\dot{x}=f(x)+g(x) u=f(x)+\sum_{i=1}^{m} u_{i} g_{i}(x)
$$

where $x \in R^{n}, u \in R^{m}$ and with $x\left(t_{0}\right)=x_{0}$, such that a cost index

$$
J=\int_{t_{0}}^{t_{f}} L(x, u, t) d t
$$

$L: R^{n} \times R^{m} \times R \rightarrow R$, is minimised.

Usually, a bound on the input amplitude is considered, so that the control must satisfy

$$
u_{\text {min }} \leq u(t) \leq u_{\text {MAX }} \quad \forall t \in\left[t_{0}, t_{f}\right]
$$

with $u_{\text {min }}, u_{M A X} \in R^{m}$.

In the definition of the problem, additional constraints can be introduced.

i. The final time instant $t_{f}$ can be fixed, so that the cost index results to be a function of the input only

$$
J(u)=\int_{t_{0}}^{t_{f}} L(x, u, t) d t
$$

Be $t_{f}^{d}$ such a prefixed value, a constraint of the form

$$
\chi\left(x\left(t_{f}\right), t_{f}\right)=0
$$

with $\chi\left(t_{f}\right)=t_{f}-t_{f}^{d}$ has to be considered, with $\chi \in C^{1}$ almost everywhere, $\operatorname{dim}\{\chi\}=1$.

ii. The final value for the state $x\left(t_{f}\right)=x_{f}$, or for $\sigma$ components only, can be fixed; in this case, $x\left(t_{f}\right)$ must satisfy a condition of the form $\chi\left(x\left(t_{f}\right)\right)=$ $x\left(t_{f}\right)-x_{f}$, with $\operatorname{dim}\{\chi\}=\sigma, 1 \leq \sigma \leq n$, and the cost index is a function of both $u$ and $t_{f}$

$$
J\left(u, t_{f}\right)=\int_{t_{0}}^{t_{f}} L(x, u, t) d t
$$

Clearly, if $t_{f}$ is left free, it has to be found along the solution computation.

The usual design procedure starts with the definition of the Hamiltonian function

$$
H(x, \lambda, u, t)=L(x, u, t)+\lambda^{T}(f(x)+g(x) u)
$$

in which the multiplier function $\lambda(t)$ is introduced, with $\lambda(t): R \rightarrow R^{n}, \lambda(t) \in C^{1}$ almost everywhere.

The Hamiltonian (7) verifies the condition

$$
H(x, \lambda, u, t)=K \quad \forall t \in\left[t_{0}, t_{f}\right]
$$

with $K \in R$. Since (7) must satisfy

$$
H\left(x, \lambda, u, t_{f}\right)=\frac{\partial \chi\left(x\left(t_{f}\right), t_{f}\right)}{\partial t_{f}} \eta
$$

for an unknown $\eta \in R, K$ is a variable to be found during the optimization procedure if the final time $t_{f}$ is fixed, with $K=\eta$, as in the previously mentioned case $i$, while it is equal to zero when the final time is left free.

From (7), and taking into account the dynamics form (1), the differential equation describing the behaviour of $\lambda$ can be obtained as

$$
\dot{\lambda}=-\left.\frac{\partial L}{\partial x}\right|^{T}-\left(\left.\frac{\partial f}{\partial x}\right|^{T}+\left.\sum_{i=1}^{m} u_{i} \frac{\partial g_{i}}{\partial x}\right|^{T}\right) \lambda
$$

under the boundary conditions

$$
\lambda\left(t_{f}\right)=-\left.\frac{\partial \chi\left(x\left(t_{f}\right), t_{f}\right)}{\partial x\left(t_{f}\right)}\right|^{T} \zeta
$$

with $\zeta \in R^{\sigma}$ to be found and $\chi$ in (5). Clearly, if constraint (5) is not present, (10) becomes

$$
\lambda\left(t_{f}\right)=0
$$

The structure of (7) suggests that if in $L(x, u, t)$ the control $u$ is present with a linear contribution, the whole Hamiltonian results to be affine with respect to the control. This property can be very useful when the minimum principle is applied.

Then, particularising the expression

$$
L(x, u, t)=\tilde{L}(x, t)+c^{T} u
$$

the structure of $L$ as in (12), used in (7), gives the compact expression

$$
\begin{aligned}
H(x, \lambda, u, t)= & \tilde{L}(x, t)+c^{T} u+\lambda^{T} f(x)+\lambda^{T} g(x) u= \\
& =\tilde{L}(x, t)+\lambda^{T} f(x)+\left(c^{T}+\lambda^{T} g(x)\right) u= \\
& =F(x, \lambda, t)+G(x, \lambda) u
\end{aligned}
$$

with

$$
\begin{aligned}
F(x, \lambda, t) & =\tilde{L}(x, t)+\lambda^{T} f(x) \\
G(x, \lambda) & =c^{T}+\lambda^{T} g(x)
\end{aligned}
$$


Clearly, the presence of the input $u$ in the cost function in a linear form is always possible, if $u_{\text {min }}$ in (3) is finite.

The minimum principle $H(x, \lambda, u, t) \leq$ $H(x, \lambda, \omega, t) \forall \omega \in\left[u_{\text {min }}, u_{M A X}\right]$ can be used, giving, for (13), the condition $G(x, \lambda) u \leq G(x, \lambda) \omega$ $\forall \omega \in\left[u_{\min }, u_{M A X}\right]$. As a consequence, the optimal control

$$
u= \begin{cases}u_{M A X} & \text { if } G(x, \lambda)<0 \\ u_{\text {min }} & \text { if } G(x, \lambda)>0\end{cases}
$$

can be obtained. The time instant $t_{s}$ such that

$$
G\left(x\left(t_{s}\right), \lambda\left(t_{s}\right)\right)=0
$$

with

$$
G\left(x^{o}\left(t_{s}^{-}\right), \lambda^{o}\left(t_{s}^{-}\right)\right) G\left(x^{o}\left(t_{s}^{+}\right), \lambda^{o}\left(t_{s}^{+}\right)\right)<0
$$

is the instant of switching in which the control changes from one extreme to the other, so getting a classical bang-bang solution. If condition (17) holds over a finite time interval

$$
G\left(x^{o}(t), \lambda^{o}(t)\right)=0 \quad t_{0} \leq t_{1} \leq t \leq t_{2} \leq t_{f}
$$

with $t_{2}>t_{1}$, then the optimal control presents a singular solution and it assumes the form

$$
u= \begin{cases}u_{M A X} & \text { if } G(x, \lambda)<0 \\ u_{s}\left(x^{o}, \lambda^{o}\right) & \text { if } G(x, \lambda)=0 \\ u_{\text {min }} & \text { if } G(x, \lambda)>0\end{cases}
$$

In this case, (18) means that $G(x(t), \lambda(t))$ is constant over a finite time interval and, then, the identities

$$
\frac{\partial^{k} G(x(t), \lambda(t))}{\partial t^{k}}=G^{(k)}(x(t), \lambda(t))=0 \forall t \in\left[t_{1}, t_{2}\right]
$$

must hold for any $k \geq 0$. Computing (20), for $k=0,1,2, \ldots$, there exists an index $r$ such that $G^{(k)}\left(x^{o}(t), \lambda^{o}(t)\right)$ is independent from $u$ if $k<r$, while, for $k=r$, the control $u$ appears explicitly. Then, the first $r$ conditions (20), for $k=0, \ldots, r-1$, give $r$ relations between state $x$ and costate $\lambda$, and from $G^{(r)}(x(t), \lambda(t))=0$ the expression for the singular control $u_{s}$ in (19) can be obtained.

Making reference to the expression (15), one has

$$
\begin{aligned}
& G^{(0)}(x, \lambda)=c^{T}+\lambda^{T} g(x)=0 \\
& G^{(1)}(x, \lambda)=\dot{\lambda}^{T} g(x)+\lambda^{T} \dot{g}(x)= \\
& =-\alpha^{T} g(x)-\lambda^{T} L_{g} f(x)-\sum_{i=1}^{m} u_{i} \lambda^{T} L_{g} g_{i}(x)+ \\
& +\lambda^{T} L_{f} g(x)+\sum_{i=1}^{m} u_{i} \lambda^{T} L_{g_{i}} g(x)= \\
& =-\alpha^{T} g(x)+\lambda^{T} a d_{f} g(x)+ \\
& +\sum_{i=1}^{m} u_{i} \lambda^{T} a d_{g_{i}} g(x)=0
\end{aligned}
$$

where the compact expressions

$$
\begin{aligned}
L_{f} g(x) & =\left(L_{f} g_{1}(x) \ldots L_{f} g_{m}(x)\right) \\
L_{g} f(x) & =\left(L_{g_{1}} f(x) \ldots L_{g_{m}} f(x)\right) \\
L_{g} g_{i}(x) & =\left(L_{g_{1}} g_{i}(x) \ldots L_{g_{m}} g_{i}(x)\right) \\
L_{g_{i}} g(x) & =\left(L_{g_{i}} g_{1}(x) \ldots L_{g_{i}} g_{m}(x)\right) \\
a d_{f} g(x) & =L_{f} g(x)-L_{g} f(x) \\
a d_{g_{i}} g(x) & =L_{g_{i}} g(x)-L_{g} g_{i}(x)
\end{aligned}
$$

are used. Note that the structures of the Lie Bracket introduced are

$$
\begin{gathered}
a d_{f} g(x)=\left(\begin{array}{llll}
a d_{f} g_{1}(x) & a d_{f} g_{2}(x) & \cdots & a d_{f} g_{m}(x)
\end{array}\right) \\
a d_{g_{i}} g(x)=\left(\begin{array}{llll}
a d_{g_{i}} g_{1}(x) & a d_{g_{i}} g_{2}(x) & \cdots & a d_{g_{i}} g_{m}(x)
\end{array}\right)
\end{gathered}
$$

recalling that $a d_{g_{i}} g_{i}(x)=0 \forall i \in[1, m]$.

If dynamics (1) is such that the vector fields $g_{i}$ commute, then $\sum_{i=1}^{m} u_{i} \lambda^{T} a d_{g_{i}} g(x)=0$, as in (Ledzewicz and Schattler, 2011; Ledzewicz et al., 2016); the identity (22) reduces to

$$
G^{(1)}(x, \lambda)=-\alpha^{T} g+\lambda^{T} a d_{f} g(x)=0
$$

and $G^{(2)}(x, \lambda)$ has to be computed, iterating the procedure. As well known (Bryson and Ho, 1969), this iteration ends at a finite index $r$ such that $G^{(r-1)}(x, \lambda)$ is not dependent from $u$ while $G^{(r)}(x, \lambda)$ is, so giving the control expression as a function of the $x$ and $\lambda$.

\section{BLOCK SUB TRIANGULAR SINGLE INPUT SYSTEMS}

The class of dynamics (1) considered is here particularised setting $x$ non negative and $m=1$. Such choices allow to simplify the notations: $m=1$ gives a simpler expression for the input contribution from (1) on, while, thanks to the non negativeness of the state, $\tilde{L}(x, t)=\alpha^{T} x$ can be used, so obtaining a fully linear term in the cost function. Then, $\dot{\lambda}$ in (10) becomes

$$
\dot{\lambda}=-\alpha-\left.\frac{\partial f}{\partial x}\right|^{T} \lambda-\left.u \frac{\partial g}{\partial x}\right|^{T} \lambda
$$

while the existence of singular solutions gives for the conditions (21) and (22) on the $G^{(k)}\left(x^{o}(t), \lambda^{o}(t)\right)$ the expressions

$$
\begin{aligned}
& G^{(0)}(x, \lambda)=c+\lambda^{T} g(x)=0 \\
& G^{(1)}(x, \lambda)=-\alpha^{T} g(x)+\lambda^{T} a d_{f} g(x)=0
\end{aligned}
$$


with (32) always independent from $u$. Then, also the computation of $G^{(2)}(x, \lambda, u)=0$ must be performed:

$$
\begin{aligned}
& G^{(2)}(x, \lambda, u)=-\alpha^{T}\left(L_{f} g(x)+u L_{g} g(x)\right)+ \\
& -\left(\alpha^{T}+\lambda^{T} \frac{\partial f}{\partial x}+u \lambda^{T} \frac{\partial g}{\partial x}\right) a d_{f} g(x)+ \\
& +\lambda^{T}\left(L_{f} a d_{f} g(x)+u L_{g} a d_{f} g(x)\right)= \\
& =\left(\lambda^{T} a d_{f}^{2} g(x)-\alpha^{T}\left(L_{f} g(x)+a d_{f} g(x)\right)\right)+ \\
& -\left(\alpha^{T} L_{g} g(x)-\lambda^{T} a d_{g} a d_{f} g(x)\right) u
\end{aligned}
$$

If $\alpha^{T} L_{g} g(x)-\lambda^{T} a d_{g} a d_{f} g(x) \neq 0$, the singular control $u_{s}$ can be obtained as

$$
u_{s}(x, \lambda)=\frac{\lambda^{T} a d_{f}^{2} g(x)-\alpha^{T}\left(L_{f} g(x)+a d_{f} g(x)\right)}{\alpha^{T} L_{g} g(x)-\lambda^{T} a d_{g} a d_{f} g(x)}
$$

otherwise $G^{(2)}(x, \lambda, u)=G^{(2)}(x, \lambda)$, the condition

$$
\lambda^{T} a d_{f}^{2} g(x)-\alpha^{T}\left(L_{f} g(x)+a d_{f} g(x)\right)=0
$$

involving $x$ and $\lambda$ only, is obtained, and a further derivative $G^{(3)}(x, \lambda, u)$ must be computed. Set $r$ as the first index such that $G^{(r)}(x, \lambda, u)$ is dependent from the input. It is easily verified that

Proposition 1. The expression of the $i-t h$ derivative $G^{(i)}(x, \lambda)$, for $i=0,1, \ldots, r-1$, is of the form

$$
G^{(i)}(x, \lambda)=\lambda^{T} a d_{f}^{i} g(x)+h_{i}(x)
$$

for suitable functions $h_{i}(x) \quad\left(h_{0}(x)=c, h_{1}(x)=\right.$ $\left.-\alpha^{T} g(x)\right)$.

Proof: It comes iteratively at $i$-th step from the structure of $G^{(i-1)}(x, \lambda)$ and the computations evidenced in (33).

The class of nonlinear dynamics considered is of the form

$$
\left(\begin{array}{c}
\dot{x}_{1} \\
\dot{x}_{2}
\end{array}\right)=\left(\begin{array}{l}
f_{1}\left(x_{1}\right) \\
f_{2}\left(x_{1}\right)
\end{array}\right)+\left(\begin{array}{l}
g_{1}\left(x_{1}\right) \\
g_{2}\left(x_{1}\right)
\end{array}\right) u
$$

where $x_{1} \in R^{r}, x_{2} \in R^{n-r}$, and the functions $f_{i}$ and $g_{i}$ defined consequently. The controllability condition

$$
\operatorname{dim}\left(\operatorname{span}\left\{g_{1}, a d_{f_{1}} g_{1}, \ldots, a d_{f_{1}}^{r-1} g\right\}\left(x_{1}\right)\right)=r
$$

for the first subsystem is assumed verified, with $x_{1}$ in a suitable domain containing $x_{1}\left(t_{0}\right)$. The example used in Section 4 to illustrate the proposed approaches fulfils this hypothesis with $r=2$.

Consequently, setting

$$
\lambda=\left(\begin{array}{l}
\lambda_{1} \\
\lambda_{2}
\end{array}\right) \quad \lambda_{1} \in R^{r}, \lambda_{2} \in R^{n-r}
$$

one has

$$
\begin{aligned}
& \frac{\partial L}{\partial x}=\left(\begin{array}{ll}
\frac{\partial L}{\partial x_{1}} & \frac{\partial L}{\partial x_{2}}
\end{array}\right) \\
& \frac{\partial f}{\partial x}=\left(\begin{array}{ll}
\frac{\partial f_{1}\left(x_{1}\right)}{\partial x_{1}} & 0 \\
\frac{\partial f_{2}\left(x_{1}\right)}{\partial x_{1}} & 0
\end{array}\right) \quad \frac{\partial g}{\partial x}=\left(\begin{array}{ll}
\frac{\partial g_{1}\left(x_{1}\right)}{\partial x_{1}} & 0 \\
\frac{\partial g_{2}\left(x_{1}\right)}{\partial x_{1}} & 0
\end{array}\right)
\end{aligned}
$$

so that (10) becomes

$$
\begin{aligned}
& \left(\begin{array}{l}
\dot{\lambda}_{1} \\
\dot{\lambda}_{2}
\end{array}\right)=-\left(\begin{array}{c}
\frac{\partial L}{\partial x_{1}} \\
\frac{\partial L}{\partial x_{2}}
\end{array}\right)+\left(\begin{array}{cc}
\frac{\partial f_{1}^{T}\left(x_{1}\right)}{\partial x_{1}} & \frac{\partial f_{2}^{T}\left(x_{1}\right)}{\partial x_{1}} \\
0 & 0
\end{array}\right) \lambda+ \\
& +u\left(\begin{array}{cc}
\frac{\partial g_{1}\left(x_{1}\right)}{\partial x_{1}} & \frac{\partial g_{2}\left(x_{1}\right)}{\partial x_{1}} \\
0 & 0
\end{array}\right) \lambda
\end{aligned}
$$

The above equation can be explicitly decomposed into

$$
\begin{aligned}
\dot{\lambda}_{1}= & -\frac{\partial L}{\partial x_{1}}-\left(\frac{\partial f_{1}^{T}\left(x_{1}\right)}{\partial x_{1}}+u \frac{\partial g_{1}\left(x_{1}\right)}{\partial x_{1}}\right) \lambda_{1}+ \\
& -\left(\frac{\partial f_{2}^{T}\left(x_{1}\right)}{\partial x_{1}}+u \frac{\partial g_{2}\left(x_{1}\right)}{\partial x_{1}}\right) \lambda_{2} \\
\dot{\lambda}_{2}= & -\frac{\partial L}{\partial x_{2}}
\end{aligned}
$$

From this structure, and on the basis of the expression of constraint (5), if present, one has the following proposition:

Proposition 2. Given the optimal control problem, with fixed time $t_{f}$ and free final conditions on the state, for a non linear dynamics of the form (36) and the cost function (4) with $L(x, u, t)=\alpha_{1}^{T} x_{1}+c u, \alpha_{1} \in R^{r}$, there exists an algorithm for the computation of the optimal singular solution which gives the expression of the control law as a pure state feedback, bringing to a bang-singular-bang optimal control, for which the singular surface can be explicitly written as a state function only. Moreover, after the last switch, the optimal control $u$ is equal to $u_{\text {min }}$.

Proof: The independence of the constraint (5) from the state $x\left(t_{f}\right)$ makes (11) hold; moreover, the choice for $L(x, u, t)$ gives, for equation (41), the simple expression $\dot{\lambda}_{2}=0$ which, for (11) and for the continuity hypothesis on $\lambda(t)$, gives $\lambda_{2}(t)=0 \forall t \in\left[t_{0}, t_{f}\right]$. This means that, for $t=t_{f}, G(x, \lambda)=c \geq 0$, and then the last condition in (19) is satisfied. Moreover, for the continuity conditions on $x$ and $\lambda$, the inequality must hold over a finite time interval, which necessarily corresponds to the last bang interval, so proving the last claim of the Proposition.

Equations $G^{(i)}(x, \lambda), i=0,1, \ldots, r-1$, can be re- 
written as

$$
\begin{aligned}
G^{(0)}(x, \lambda) & =\lambda_{1}^{T} g_{1}\left(x_{1}\right)+c=0 \\
G^{(1)}(x, \lambda) & =\lambda_{1}^{T} a d_{f_{1}} g_{1}\left(x_{1}\right)-\alpha_{1}^{T} g_{1}\left(x_{1}\right)=0 \\
\cdots & =\ldots \\
G^{(r-1)}(x, \lambda) & =\lambda_{1}^{T} a d_{f_{1}}^{r-1} g_{1}\left(x_{1}\right)+h_{r-1}\left(x_{1}\right)=0
\end{aligned}
$$

or in the compact form

$$
\begin{aligned}
& \lambda_{1}^{T}\left(g_{1}\left(x_{1}\right), \quad a d_{f_{1}} g_{1}\left(x_{1}\right), \quad \ldots \quad a d_{f_{1}}^{i-1} g_{1}\left(x_{1}\right)\right)= \\
& =\left(\begin{array}{llll}
-c, & \alpha_{1}^{T} g_{1}\left(x_{1}\right), \quad \ldots & h_{i-1}\left(x_{1}\right)
\end{array}\right)
\end{aligned}
$$

from which

$$
\lambda_{1}^{T}=\left(-c, \ldots h_{i-1}\left(x_{1}\right)\right)\left(g_{1}\left(x_{1}\right), \ldots a d_{f_{1}}^{i-1} g_{1}\left(x_{1}\right)\right)^{-1}
$$

can be computed under the controllability condition (37). Then, the costate $\lambda$ is fully known, at least as a function of the state. A first consequence of this fact is the possibility to express the singular control $u_{s}(x, \lambda)$ as a state function only, in particular a function of $x_{1}$, $u_{s}=u_{s}\left(x_{1}\right)$.

Now, from (8), applied in (13) and then in (14) when (18) holds, one gets the expression

$$
F(x, \lambda, t)=\alpha_{1}^{T} x_{1}+\lambda_{1}^{T} f_{1}\left(x_{1}\right)=K
$$

which, using (45), brings to the state function

$$
\begin{gathered}
K=\alpha_{1}^{T} x_{1}+ \\
+\left(-c \ldots h_{i-1}\left(x_{1}\right)\right)\left(g_{1}\left(x_{1}\right) \ldots a d_{f_{1}}^{i-1} g_{1}\left(x_{1}\right)\right)^{-1} f_{1}\left(x_{1}\right)
\end{gathered}
$$

which fully describes the singular surface in the $x_{1}$ subspace. As far as the subspace $x_{2}$ is concerned, once the evolution of $x_{1}$ is determined, also $x_{2}$ is fully known, since (36), the singular control $u_{s}\left(x_{1}\right)$ and the evolution of $x_{1}$ satisfying (47) allow to compute $x_{2}(t)$ $\forall t \in\left[t_{1}, t_{2}\right]$. The value of the unknown parameter $K$ can be computed noting that, from (13) evaluated af $t=t_{f}$, one has

$$
H\left(x, \lambda, u, t_{f}\right)=\alpha_{1}^{T} x_{1}\left(t_{f}\right)=K
$$

being $u\left(t_{f}\right)=0$ as well as $\lambda\left(t_{f}\right)=0$. Expression (48), once $x\left(t_{f}\right)$ is computed, gives $K$.

Proposition 2 allows to fully compute the singular part of the optimal solution. The full solution (19) is obtained including the conditions in (16).

Proposition 3. Given the optimal control problem, with fixed time $t_{f}$ and fixed final condition on the state $x\left(t_{f}\right)=x_{f}$, for a non linear dynamics of the form (36) and the cost function (4) with $L(x, u, t)=\alpha_{1}^{T} x_{1}+c u$, $\alpha_{1} \in R^{r}$, there exists an algorithm for the computation of the optimal singular solution which gives the expression of the control law as a pure state feedback, bringing to a bang-singular-bang optimal control, for which the singular surface can be explicitly written as a state function only.

Proof: The presence of constraint (5) does not allow to know $\lambda\left(t_{f}\right)$ due to expression (10). However, $\dot{\lambda}_{2}=$ 0 is still true and then one has $\lambda_{2}(t)=\lambda_{2}\left(t_{f}\right)=$ const. In this case equations (42), (43), (44) assume the form

$$
\begin{aligned}
G^{(0)}(x, \lambda) & =\lambda_{1}^{T} g_{1}\left(x_{1}\right)+\lambda_{2}^{T}\left(t_{f}\right) g_{2}\left(x_{1}\right)=0 \text { (49) } \\
G^{(1)}(x, \lambda) & =\lambda_{1}^{T} a d_{f_{1}} g_{1}\left(x_{1}\right)-\alpha_{1}^{T} g_{1}\left(x_{1}\right)+ \\
& +\lambda_{2}^{T}\left(t_{f}\right)\left[f_{2}, g_{2}\right]\left(x_{1}\right)=0 \\
\ldots & =\ldots \\
G^{(r-1)}(x, \lambda) & =\lambda_{1}^{T} a d_{f_{1}}^{r-1} g_{1}\left(x_{1}\right)+h_{r-1}\left(\lambda_{2}\left(t_{f}\right), x_{1}\right)
\end{aligned}
$$

The same considerations as in the proof of Proposition (2) can be carried on, computing $\lambda_{1}$ as in (45), whose expression in this case is $\lambda_{1}\left(\lambda_{2}\left(t_{f}\right), x_{1}\right)$. Both the singular control $u_{s}$ and the singular surface (47) can be computed, as in the proof of Proposition 2, as function of $x_{1}$ but they results to be parametrised by $\lambda_{2}\left(t_{f}\right)$. The same follows for $x_{2}(t)$. However, the imposition of condition (5) allows to determine the actual values for $\lambda_{2}\left(t_{f}\right)$, so getting again the full computation of the singular solution $u_{s}=u_{s}\left(x_{1}\right)$ and of the singular state space surface. The full knowledge of all the state and control variables at $t=t_{f}$ allows to obtain the value $K$ from the evaluation of (13) at the final time, as for the previous Proposition.

The last case here addressed refers to a free time fixed final conditions on the state variables. It is possible to state the following

Proposition 4. Given the optimal control problem, with free final time $t_{f}$ and the constraint (5) for the final conditions on the state, for a non linear dynamics of the form (36) and the cost function (4) with $L(x, u, t)=\alpha_{1}^{T} x_{1}+c u, \alpha_{1} \in R^{r}$, there exists an algorithm for the computation of the optimal singular solution which gives the expression of the control law as a pure state feedback, bringing to a bang-singularbang optimal control, for which the singular surface can be explicitly written as a state function only.

Proof: The proof comes straightforwardly from the one of Proposition 3, since the only difference is in the fact that being the final time $t_{f}$ free, $K$ in (8) and in all the derived expressions, as far as (47), is equal to zero. All the other considerations still hold.

The procedure is illustrated in next Section making reference to a classical SIR epidemic diffusion, for which several optimal control approaches can be found in literature. 


\section{EXAMPLE}

With reference to the general formulation introduced in Section 2, it is now considered the classical mathematical model describing the SIR epidemic spread

$$
\begin{aligned}
\dot{S} & =-\beta S I-S u+\mu \\
\dot{I} & =\beta S I-\gamma I \\
\dot{R} & =\gamma I
\end{aligned}
$$

with given initial conditions $S_{0}, I_{0}, R_{0}$, and box constraints $u(t) \in\left[0, u_{M A X}\right]$, for which the control law $u(t)$ minimising the cost function

$$
J(S(t), I(t), R(t), u(t))=\int_{t_{0}}^{t_{f}}(a I(t)+c u(t)) d t
$$

has to be found. $t_{f}$ is assumed fixed. The aim of the control action is to minimize the number of infected subjects $I$ in the fixed time interval $\left[0, t_{f}\right]$ by using as less resources as possible; the positive parameters $a$ and $c$ represent the weights of these two contrasting requirements.

Setting

$$
x=\left(\begin{array}{l}
x_{1} \\
x_{2}
\end{array}\right) \quad \text { with } \quad x_{1}=\left(\begin{array}{l}
S \\
I
\end{array}\right) \quad \text { and } \quad x_{2}=R
$$

the formulation corresponds to the case considered in Proposition 2, with $r=2, \alpha_{1}^{T}=\left(\begin{array}{ll}0 & a\end{array}\right), \alpha^{T}=$ $\left(\begin{array}{ll}\alpha_{1}^{T} & 0\end{array}\right)$ and

$$
\begin{array}{ll}
f_{1}\left(x_{1}\right)=\left(\begin{array}{c}
-\beta S I+\mu \\
\beta S I-\gamma I
\end{array}\right) & g_{1}\left(x_{1}\right)=\left(\begin{array}{c}
-S \\
0
\end{array}\right) \\
f_{2}\left(x_{1}\right)=\gamma I & g_{2}\left(x_{1}\right)=0
\end{array}
$$

Introducing

$$
\lambda=\left(\begin{array}{l}
\lambda_{1} \\
\lambda_{2}
\end{array}\right) \quad \text { with } \quad \lambda_{1} \in R^{2} \quad \text { and } \quad \lambda_{2} \in R
$$

the Hamiltonian (13) can be written as

$$
\begin{aligned}
H(x, \lambda, u)= & \alpha_{1}^{T} x_{1}+c u+\lambda_{1}^{T} f_{1}\left(x_{1}\right)+\lambda_{2} f_{2}\left(x_{1}\right)+ \\
& +\lambda_{1}^{T} g_{1}\left(x_{1}\right) u= \\
= & \alpha_{1}^{T} x_{1}+\lambda_{1}^{T} f_{1}\left(x_{1}\right)+\lambda_{2} f_{2}\left(x_{1}\right)+ \\
& +\left(c+\lambda_{1}^{T} g_{1}\left(x_{1}\right)\right) u= \\
= & F(x, \lambda)+G(x, \lambda) u
\end{aligned}
$$

with

$$
\begin{aligned}
& F(x, \lambda)=\alpha_{1}^{T} x_{1}+\lambda_{1}^{T} f_{1}\left(x_{1}\right)+\lambda_{2} f_{2}\left(x_{1}\right) \\
& G(x, \lambda)=c+\lambda_{1}^{T} g_{1}\left(x_{1}\right)
\end{aligned}
$$

and for which

$$
H(x, \lambda, u)=K \quad \forall t \in\left[t_{0}, t_{f}\right], \quad K \in R
$$

The costate dynamics (40) and (41) for $\lambda$ can be computed, getting

$$
\begin{aligned}
\dot{\lambda}_{1}= & -\left(\begin{array}{l}
0 \\
a
\end{array}\right)-\left(\begin{array}{cc}
-\beta I & \beta I \\
-\beta S & \beta S-\gamma
\end{array}\right) \lambda_{1}+ \\
& -u\left(\begin{array}{cc}
-1 & 0 \\
0 & 0
\end{array}\right) \lambda_{1}-\left(\begin{array}{l}
0 \\
\gamma
\end{array}\right) \lambda_{2} \\
\dot{\lambda}_{2}= & 0
\end{aligned}
$$

with $\lambda\left(t_{f}\right)=0$ from the free final state $x$ condition. Then, as expected, from (64) one has $\lambda_{2}(t)=0$.

The computation of $a d_{f_{1}} g_{1}\left(x_{1}\right)$ yields

$$
a d_{f_{1}} g_{1}\left(x_{1}\right)=\left(\begin{array}{c}
\beta S I-\mu \\
0
\end{array}\right)-\left(\begin{array}{c}
\beta S I \\
-\beta S I
\end{array}\right)=\left(\begin{array}{c}
-\mu \\
\beta S I
\end{array}\right)
$$

so that expressions (49) and (50) assume the form

$$
\begin{aligned}
& G^{(0)}(x, \lambda)=c+\lambda_{1}^{T}\left(\begin{array}{c}
-S \\
0
\end{array}\right)=0 \\
& G^{(1)}(x, \lambda)=\lambda_{1}^{T}\left(\begin{array}{c}
-\mu \\
\beta S I
\end{array}\right)=0
\end{aligned}
$$

from which the variable $\lambda_{1}$ can be computed, as a function of $x_{1}=\left(\begin{array}{ll}S & I\end{array}\right)^{T}$, obtaining

$$
\lambda_{1}=\left(\begin{array}{cc}
-S & 0 \\
-\mu & \beta S I
\end{array}\right)^{-1}\left(\begin{array}{c}
-c \\
0
\end{array}\right)=\left(\begin{array}{c}
\frac{c}{S} \\
\frac{\mu c}{\beta S^{2} I}
\end{array}\right)
$$

The expression for $G^{(2)}(x, \lambda)$ can be computed as

$$
\begin{aligned}
G^{(2)}(x, \lambda)= & \lambda_{1}^{T} a d_{f_{1}}^{2} g_{1}\left(x_{1}\right)-\alpha_{1}^{T} a d_{f_{1}} g_{1}\left(x_{1}\right)+ \\
& +u \lambda_{1}^{T} a d_{g_{1}} a d_{f_{1}} g_{1}\left(x_{1}\right)
\end{aligned}
$$

from which the singular control

$$
u_{s}(x, \lambda)=\frac{\lambda_{1}^{T} a d_{f_{1}}^{2} g_{1}\left(x_{1}\right)-\alpha_{1}^{T} a d_{f_{1}} g_{1}\left(x_{1}\right)}{\lambda_{1}^{T} a d_{g_{1}} a d_{f_{1}} g_{1}\left(x_{1}\right)}
$$

is obtained. Computing

$$
\begin{gathered}
a d_{f_{1}}^{2} g_{1}\left(x_{1}\right)=\left(\begin{array}{c}
-\beta \mu I+\beta^{2} S^{2} I \\
-\beta^{2} S I^{2}+2 \beta \mu I
\end{array}\right) \\
a d_{g_{1}} a d_{f_{1}} a d_{g_{1}}\left(x_{1}\right)=\left(\begin{array}{c}
-\mu \\
-\beta S I
\end{array}\right)
\end{gathered}
$$

in (70), and making use of (68), the state feedback singular control

$$
\begin{gathered}
u_{s}(x)=\frac{a \beta S I-\beta^{2} c S I+\frac{2 \beta c \mu I}{S}-\frac{2 c \mu^{2}}{S^{2}}}{-2 \frac{c \mu}{S}}= \\
=\beta I\left(\frac{\beta c-a}{2 c \mu} S^{2}-1\right)+\frac{\mu}{S}
\end{gathered}
$$

is computed. Then, the full state feedback control law (19) is obtained. 
From (60), according to (46) and (47), one has for the singular surface

$$
\begin{aligned}
& \left(\begin{array}{ll}
0 & a
\end{array}\right)\left(\begin{array}{l}
S \\
I
\end{array}\right)+\left(\begin{array}{ll}
\frac{c}{S} & \frac{\mu c}{\beta S^{2} I}
\end{array}\right)\left(\begin{array}{c}
-\beta S I+\mu \\
\beta S I-\gamma I
\end{array}\right)= \\
& a I-\beta c I+2 \frac{c \mu}{S}-\frac{c \gamma \mu}{\beta S^{2}}=K
\end{aligned}
$$

Finally, from (48),

$$
K=a I\left(t_{f}\right)
$$

The time history of the bang-singular-bang control is depicted in Figure 1, where the time instant $t=5.32$ in which it switches from the constant value $u_{M A X}=1$ to $u_{s}$, and the time $t=15.3$, in which it switches from $u_{s}$ to $\min =0$ are evidenced by the two vertical dotted lines. The corresponding state evolution is represented in Figure 2 for the $x_{1}-x_{2}$ plane projection: the initial part of the trajectory from the starting conditions $x_{1,0}=50$ and $x_{2,0}=10$, the squared point, to the intersection with the singular curve (dotted line), denoted by a dashed curve, represents the evolution under the upper bound value for the control. Then, under the singular control (73), the trajectory follows the singular curve along the solid arc, until the second switch from $u=u_{s}$ to $u=u_{\min }=0$ occurs, yielding to the free evolution from the singular arc to the final point (diamond marker).

The simulation has been performed setting $t_{f}=$ 20 , fixing the model parameters to $\beta=0.01, \gamma=0.4$ and $\mu=10$ and choosing the weights $\alpha=1$ and $c=1$ in the cost function (55).

\section{CONCLUSIONS}

Sufficient conditions under which the solution of a singular optimal control problem can be directly expressed as a state feedback law are provided, allowing its computation by means of a simple forward integration of the system dynamics only. The class addressed can be enriched, preserving the results, requiring the introduction of some integrability properties for triangular and sub triangular structure; this analysis is the object of a forthcoming work.

\section{ACKNOWLEDGEMENTS}

This work was supported by Sapienza University of Rome, Grants No. RP11715C82440B12 and No. 191/2016.

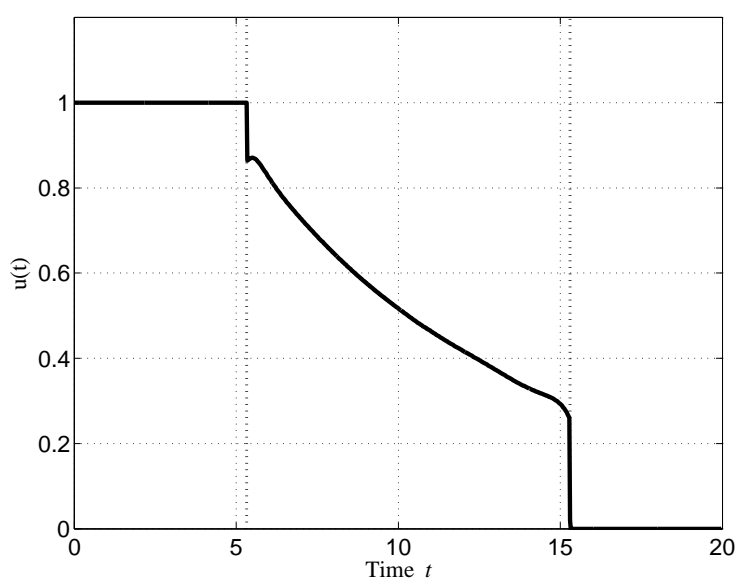

Figure 1: The Bang-Singular-Bang optimal control $u(t)$.

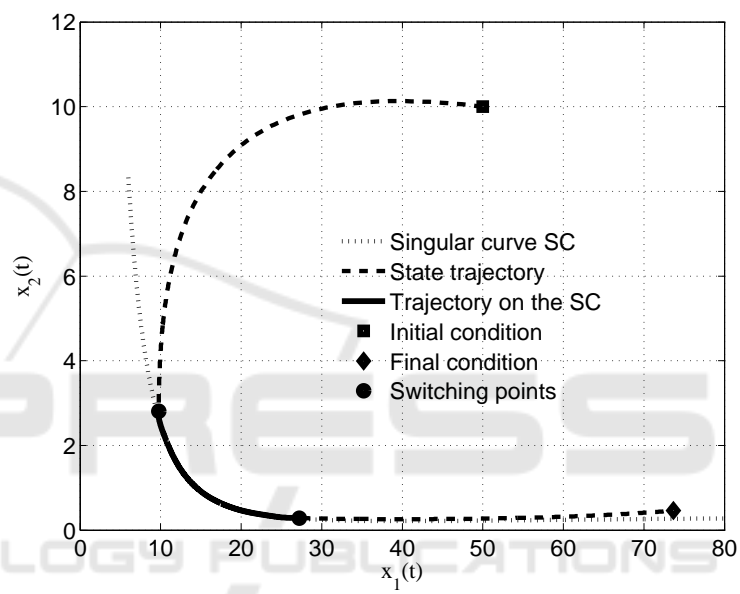

Figure 2: State trajectory in the $x_{1}-x_{2}$ plane, compared with the singular curve.

\section{REFERENCES}

Athans, M. and Falb, P. (1996). Optimal Control. McGrawHill, Inc., New York.

Bakare, E., Nwagwo, A., and Danso-Addo, E. (2014). Optimal control analyis of an sir epidemic model with constant recruitment. International Journal of Applied Mathematical Research, 3.

Behncke, H. (2000). Optimal control of deterministic epidemics. Optimal control applications and methods, 21.

Bryson, A. and Ho, Y. (1969). Applied optimal control: optimization, estimation, and control.

Di Giamberardino, P., Compagnucci, L., Giorgi, C. D., and Iacoviello, D. (2018). Modeling the effects of prevention and early diagnosis on hiv/aids infection diffusion. IEEE Transactions on Systems, Man and Cybernetics: Systems.

Di Giamberardino, P. and Iacoviello, D. (2017). Optimal control of SIR epidemic model with state dependent 
switching cost index. Biomedical Signal Processing and Control, 31 .

Fraser-Andrews, G. (1989). Finding candidate singular optimal controls: a state of art survey. Journal of Optimization Theory and Applications, 60.

Hartl, R., Sethi, S., and Vickson, R. (1995). A survey of the maximum principles for optimal control problems with state constraints. Society for Industrial and Applied Mathematics, 37:181-218.

Johnson, C. and Gibson, J. (1963). Singular solutions in problems of optimal control. IEEE Trans. on Automatic Control, 8(1):4-15.

Ledzewicz, U., Aghaee, M., and Schattler, H. (2016). Optimal control for a sir epidemiological model withtimevarying population. 2016 IEEE Conference on Control Applications.

Ledzewicz, U. and Schattler, E. (2011). On optimal singular controls for a general SIR-model with vaccination and treatment. Discrete and continuous dynamical systems.

Vossen, G. (2010). Switching time optimization for bangbang and singular controls. Journal of Optimization Theory and Applications, 144. 